\title{
Coarctation of the aorta associated with a sinus venosus atrial septal defect presenting with endocarditis in middle age
}

\author{
D.F. D'Costa and A.R. Davidson
}

Department of Medicine, General Hospital, Kettering, Northants NN16 8UZ, UK

\begin{abstract}
Summary: We report the case of a 44 year old man who presented with an infective endocarditis and was found to have a coarctation of the aorta associated with a sinus venosus atrial septal defect and a small ventricular septal defect. The association of coarctation with a sinus venous atrial septal defect presenting with endocarditis in an adult is extremely rare.
\end{abstract}

\section{Introduction}

It is uncommon for coarctation of the aorta to present in middle age. The association of an atrial septal defect (ASD) with coarctation has been recognized in infants but not adults. ${ }^{1,2} \mathrm{~A}$ single case of a secundum type of ASD in association with slight coarctation in a 38 year old adult was reported. ${ }^{3}$ We report a case of coarctation with a sinus venosus ASD and a small ventricular septal defect (VSD) - presenting for the first time in middle age with a bacterial endocarditis.

\section{Case report}

A 44 year old male presented with a 4-month history of malaise, a weight loss of about 7 kilograms and occasional night sweats. A month earlier he had an uncomplicated inguinal hernia repair. On examination, he was apyrexial. He had a sinus tachycardia of 120 beats per minute with a radio-femoral delay. The blood pressure in the right arm was $170 / 85 \mathrm{mmHg}$ and the left arm $150 / 80 \mathrm{mmHg}$. The blood pressure in the legs was unrecordable. He had a normal first heart sound, an ejection systolic murmur down the left sternal edge, a split second sound and a diastolic murmur down the left sternal edge. He had loud bruits over the scapulae. There were no signs of failure. The rest of the examination was normal.

Investigations were as follows: haemoglobin $13.5 \mathrm{~g} / \mathrm{dl}$, white cell count $10,000 / \mathrm{mm}^{3}$, erythrocyte sedimentation rate $40 \mathrm{~mm} / \mathrm{h}$. The electrocardio-

Correspondence: D.F. D'Costa, M.R.C.P., Leicester General Hospital, Gwendolen Road, Leicester LE5 4PW, UK

Accepted: 25 April 1990 gram showed atrial flutter with $4: 1$ block and a rate of 75/minute. Chest X-ray (see Figure 1) revealed bilateral rib notching and an increased cardiothoracic ratio. There was a double aortic knuckle and the main pulmonary artery was enlarged with pulmonary plethora present. The appearances suggested a left to right shunt and coarctation of the aorta. Blood cultures grew Streptococcus faecalis and Streptococcus sanguis. An echocardiogram showed sinus venosus ASD, aortic coarctation and aortic regurgitation. No vegetations were visible. Cardiac catheterization gave the following results: oxygen saturation: superior vena cava $70.5 \%$, right atrium (RA) $83 \%$, right ventricle (RV) $\mathbf{9 0 . 5 \%}$.

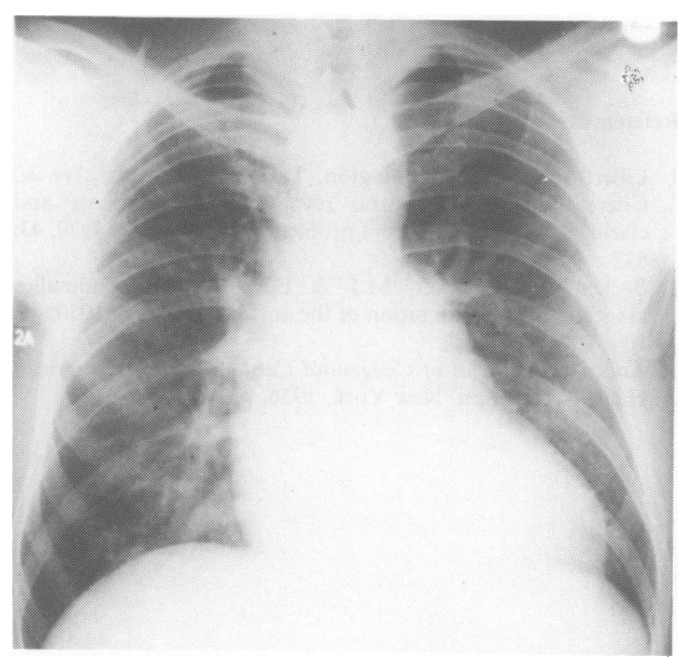

Figure 1 Coarctation of the aorta (rib-notching) with enlarged pulmonary artery and pulmonary plethora (indicating left-to-right shunt). 
Pressures were: RA mean 4, RV 55/1, pulmonary artery $45 / 10$ mean 20 , left atrium 5 , left ventricle $160 / 2$, ascending aorta $160 / 70$, descending aorta $100 / 70$. The pulmonary:systemic flow ratio was 2.6:1. A magnetic resonance imaging scan showed a coarctation of the aorta with aortic regurgitation, sinus venosus ASD with anomalous pulmonary venous drainage, a small sub-tricuspid VSD and dilated ventricles with a normal ejection fraction. A dental X-ray showed an abscess under the left upper first incisor, which was extracted. He was treated with intravenous antibiotics (penicillin and gentamicin) for 6 weeks and successfully cardioverted to sinus rhythm. A patch angioplasty repair of the coarctation was carried out 3 months later using the internal mammary artery. During the operation a fairly large aneurysm of one of the intercostal arteries was noted with adherence to the medial side of the aorta and gross vascularity suggesting an endarteritis. He required a further cardioversion post-operatively and has since remained in sinus rhythm. At follow-up over the year, he has remained well, having regained his weight. The infection was probably on the VSD as there was no infection found on the coarctation during the operation and infections on the ASD are extremely rare.

\section{Discussion}

Approximately $55 \%$ of patients with coarctation of the aorta present in the first month of life and $80 \%$ by the first year. ${ }^{4}$ It is uncommon for coarctation to present in adults. The delay in diagnosis may be related to cases being undetected by paediatricians and other physicians despite physical findings. ${ }^{5}$
Additionally a coarctation with any other associated anomaly tends to present much earlier on in life and has a very high mortality. ${ }^{6}$ It is hence surprising that this patient who had associated anomalies of an ASD and VSD presented so late in life.

In a review of 234 patients with coarctation of the aorta (age ranges 1 day to 72 years) between 1948 and 1978, Liberthson et al. showed that in $34 \%$ of the patients additional cardiac anomalies such as patent ductus ateriosus, VSD, aortic stenosis and insufficiency and rheumatic mitral valve disease were present. ${ }^{1}$ There were no cases associated with ASD. Becker's series, with particular reference to infancy, showed numerous anomalies associated with coarctation of the aorta, of which ASD formed $13 \%{ }^{2}$

The patient presented with a bacteraemic illness from a dental abscess and was found to have a coarctation. This was associated with an ASD and an anomalous pulmonary vein, i.e., sinus venosus type ASD. He had a successful repair of the coarctation. In view of the pulmonary:systemic flow ratio being greater than $2: 1$, he is being offered a surgical correction of his ASD. Interestingly, he had a hernia operation a month before presentation and had an uneventful recovery. He received no antibiotics at that admission as his cardiac abnormality was not known. A chest X-ray was not done at that stage. That coarctation with associated anomalies can still present in adults with an endocarditis is of great importance.

Coarctation associated with a sinus venosus ASD presenting in adults has not been documented before, presumably because patients present early on in life.

\section{References}

1. Liberthson, R.R., Pennington, D.G., Jacobs, M.L. et al. Coarctation of the aorta: review of 234 patients and clarification of management problems. Am J Cardiol 1979, 43: 837.

2. Becker, A.E., Becker, M.J. \& Edwards, J.E. Anomalies associated with coarctation of the aorta. Circulation 1970, 41: 1067-1076.

3. Abbot, M.E. Atlas of Congenital Cardiac Disease. American Heart Association, New York, 1936, pp. 33-35.
4. Shinebourne, E.A., Tam, A.S.Y., Elseed, A.M. et al. Coarctation of the aorta in infancy and childhood. Br Heart J 1976, 38: 375.

5. Strafford, M.A., Griffiths, S.P. \& Gersony, W.M. Coarctation of the aorta: a study in delayed detection. Pediatrics 1982, 69: 159-163.

6. Sinha, S.N., Kardatzke, M.L., Cole, R.B. et al. Coarctation of the aorta in infancy. Circulation 1969, 40: 305. 